\title{
ON THE INTEGRATION PROCESSES OF A. HUŤA
}

\author{
J. C. BUTCHER
}

(received 17 August 1962)

Huta [1], [2] has given two processes for solving a first order differential equation

$$
y^{\prime}=f(x, y)
$$

to sixth order accuracy. His methods are each eight stage Runge-Kutta processes and differ mainly in that the later process has simpler coefficients occurring in it.

In the derivation of the coefficients in these processes, the Taylor expansions for the true solution and for the approximation generated by the Runge-Kutta process are compared and it is found that for sixth order accuracy, 31 separate conditions must be satisfied. For a $\nu$ stage RungeKutta process, there are $\frac{1}{2} \nu(\nu+1)$ parameters to be chosen so the value $v=8$ (giving 36 parameters) is used.

However, if the analysis had been made in terms of a set of simultaneous first order equations

$$
y^{\prime}=f(y)
$$

there would have been 37 conditions to satisfy and one would have expected a higher value of $v$ to be required. It is remarkable that, in fact, Huta's processes are applicable to a set of simultaneous equations. In this note we shall show that the remaining six conditions are satisfied.

In table 1 the correspondence is shown between the terms used in the expansions of (1) and of (2). The notation for the single equation is Huta's $[1]^{*}$, whereas that for the general set of equations is as described in [3].

* Huta's symbolism may be summarized as follows:

$$
\begin{aligned}
f_{1} & =\frac{\partial^{\prime} f}{\partial y^{i}}, \\
D^{(n)} f_{6} & =\sum_{j=0}^{n} \frac{n !}{j !(n-j) !} f^{s} \frac{\partial^{n+i} f}{\partial x^{n-j} \partial y^{i+j}}, \\
D f_{1} & =D^{(1) f_{1}} . \\
D^{(n)} f & =D^{(n)} f_{0}, \\
D f & =D f_{0} .
\end{aligned}
$$


The value $r$ in the first column is the order of the corresponding elementary differential.

TABLE 1

\begin{tabular}{|c|c|c|}
\hline$r$ & Term in (1) & Term in (2) \\
\hline 1 & $t$ & $t$ \\
\hline 2 & $D f$ & $\{\boldsymbol{f}\}$ \\
\hline 3 & $D^{(2)} t$ & $\left\{\boldsymbol{r}^{\boldsymbol{n}}\right\}$ \\
\hline $\begin{array}{l}3 \\
4\end{array}$ & $\begin{array}{l}f_{1} D f \\
D^{(x)} f\end{array}$ & $\begin{array}{l}\{,\}_{2} \\
\left\{f^{0}\right\}\end{array}$ \\
\hline 4 & $D f_{1} D f$ & $\{\{f\}\}$ \\
\hline 4 & $f_{1} D^{(2)} f$ & $\left\{f^{f 2}\right\}_{2}$ \\
\hline 4 & $f_{1}^{2} D f$ & $\{a\}_{a}$ \\
\hline 5 & $D^{(a)} f$ & $\{\boldsymbol{H}\}$ \\
\hline 5 & $D^{(2)} f_{1} D f$ & $\left\{\{f\} f^{2}\right\}$ \\
\hline 5 & $f_{2}(D f)^{2}$ & $\left\{\left\{n^{2}\right\}\right.$ \\
\hline 6 & $D f_{1} D^{(s)} f$ & $\left.\left\{\left\{f^{2}\right\}\right)\right\}$ \\
\hline 5 & $f_{1} D f_{1} D f$ & $\left.\left\{\left\{f_{2}\right\}\right\}_{2} f\right\}$ \\
\hline 5 & $f_{1} D^{(s)} f$ & $\left\{f^{2}\right\}^{8}$ \\
\hline 5 & $t_{1}^{2} D^{(2)} f$ & $\left\{f^{2}\right\}_{8}$ \\
\hline 6 & $f_{1}^{2}=D f$ & $\left\{n_{4}\right.$ \\
\hline 6 & $D^{(b)} f$ & $\left\{f^{5}\right\}$ \\
\hline 6 & $D^{(*)} f_{1} D f$ & $\left\{\{\boldsymbol{f}\} \boldsymbol{F}^{\mathbf{s}}\right\}$ \\
\hline 6 & $D f_{2}(D f)^{2}$ & $\left\{\{\}^{2} f\right\}$ \\
\hline 6 & $D^{(8)} f_{1} D^{(2)} f$ & $\left\{\left\{\boldsymbol{r}^{n}\right\} \boldsymbol{f}^{\boldsymbol{n}}\right\}$ \\
\hline 6 & $f_{1} D / D^{(2)} f_{3}$ & $\begin{array}{c}\left\{\left\{f_{1}\right\}_{2} f^{2}\right\} \\
\left\{\left\{f\left\{f^{2}\right\}\right.\right.\end{array}$ \\
\hline 6 & $f_{a} D f D^{(2)} f$ & $\left\{\left\{f^{2}\right\}\{f\}\right\}$ \\
\hline 6 & $f_{1} f_{2}(D f)^{2}$ & $\left\{\begin{array}{l}\left\{\left\{_{2} f\right\}_{2}\{f\}\right\} \\
\left\{f_{2}\left\{f_{2}\right\}\right.\end{array}\right.$ \\
\hline 6 & $D f_{1} D^{(3)} f$ & $\begin{array}{l}\left\{\{\{\}\}_{2}\right. \\
\{\{P\} f\}\end{array}$ \\
\hline 6 & $D f\left(D f_{2}\right)^{2}$ & $\{\{\{\} \cap\})$ \\
\hline 6 & $f_{1} D f_{1} D^{(s)} f$ & $\left\{\begin{array}{l}\left\{\left\{_{2} f^{2}\right\}_{2} f\right\} \\
\left\{_{2}\left\{f^{2}\right\}\right\}_{2}\end{array}\right.$ \\
\hline 6 & $f_{1}^{2} D f D f_{1}$ & $\left\{\begin{array}{l}\left.\left\{{ }_{3} f\right\}_{2} f\right\} \\
\left\{2\{\}_{2} f\right\}_{8}\end{array}\right.$ \\
\hline 6 & $f_{2} D^{(1)} f$ & $\left\{f^{4}\right\}_{3}$ \\
\hline 6 & $f_{1}^{2} D^{(x)} f$ & $\left\{{ }_{3} f^{3}\right\}_{3}$ \\
\hline 6 & $f_{3}{ }^{3} D^{(2)} f$ & $\left\{\mathbf{a}^{2}\right\}_{4}$ \\
\hline 6 & $f_{1}<D f$ & $\left\{f_{5}\right\}_{5}$ \\
\hline
\end{tabular}

It was shown in [3] that to each elementary differential $F$, there corresponds an elementary weight $\Phi$ (a polynomial in the parameters of the Runge-Kutta process) and an integer $\gamma$ such that, the equation for agreement of terms in $F$ in the true and in the approximate expansion is

$$
\Phi=1 / \gamma .
$$

In all that follows, when an elementary weight is represented by $\Phi, \Phi^{\prime}$, 
$\Phi^{\prime \prime}, \Phi_{1}, \Phi_{2}, \cdots$, then $\gamma, \gamma^{\prime}, \gamma^{\prime \prime}, \gamma_{1}, \gamma_{2}, \cdots$ will represent the corresponding integral constants; $r, r^{\prime}, r^{\prime \prime}, r_{1}, r_{2}, \cdots$ will represent the corresponding orders and $\boldsymbol{F}, \boldsymbol{F}^{\prime}, \boldsymbol{F}^{\prime \prime}, \boldsymbol{F}_{1}, \boldsymbol{F}_{2}, \cdots$ will represent the corresponding elementary differentials.

We see from table 1 , for example, that if $F_{1}=\left\{\left\{_{2} f\right\}_{2} f\right\}, F_{2}=\left\{_{2}\{f\}\right\}_{2}$, then these terms, each of order 5 , correspond to the same term, $f_{1} D f_{1} D f$ in Huta's expansion. Thus instead of the two conditions

$$
\Phi_{1}=1 / \gamma_{1}, \quad \Phi_{2}=1 / \gamma_{2}
$$

Huta finds a single condition

$$
\Phi_{1}+\Phi_{2}=1 / \gamma_{1}+1 / \gamma_{2} .
$$

A similar situation exists for the other grouped terms as well; instead of all 37 conditions of the form (3) being imposed, 26 such conditions are used while 4 further conditions are given by linear combinations of pairs of such equations. Finally, a linear combination of three of these equations is used making a total of 31 conditions. These are equations $\left(\mathbf{l}^{\prime}\right)-\left(31^{\prime}\right)$ in $[\mathbf{l}]$.

To show that the complete set of $\mathbf{3 7}$ conditions is actually satisfied in Huta's solutions, we need only verify that one of each pair and two of the three grouped conditions hold. We will verify (3) for the following cases

$$
\begin{aligned}
& {\left[_{2}[\phi] \phi\right]_{2},} \\
& \left.{ }_{2}[\phi] \phi^{2}\right]_{2}, \\
& {\left[{ }_{2}[\phi]^{2}\right]_{2},} \\
& \left.{ }_{2}\left[\phi^{2}\right] \phi\right]_{2}, \\
& \left.{ }_{2}\left[{ }_{2} \phi\right]_{2} \phi\right]_{2}, \\
& \left.{ }_{3}[\phi] \phi\right]_{3} .
\end{aligned}
$$

However, it is first necessary to show that, using the notation of [3] with $\nu=8$ and $c_{i}$ defined as $\sum_{j=1}^{8} a_{i j}$ for $i=1,2, \cdots, 8$,

$$
\sum_{i=1}^{8} b_{i} a_{i j}=b_{j}\left(1-c_{j}\right)
$$

for each value of $j(j=1,2, \cdots, 8)$.

For $j=2,3, \cdots, 7 ;(10)$ is equivalent in Huta's notation to

$$
z_{j-1}=p_{j-1}\left(1-\phi_{j-1}\right)
$$

and for $j=3,4, \cdots, 7$ he shows $([1]$, p. 219) that with his processes this is satisfied.

For $j=2$, he has shown $z_{1}=0$ and as he assumes $p_{1}=0$ this case is also covered. For $j=8$ 


$$
\sum_{i=1}^{8} b_{i} a_{i j}=0
$$

as each $a_{i j}$ occurring in this sum is zero. Moreover, since the value $\phi_{7}=c_{8}=1$ is chosen $(p 220),(10)$ is true for this case as well. It remains to prove $(10)$ for the remaining case $j=1$. This is easily seen from the fact that the sums of the two sides of $(10)$ for $j=1,2, \cdots, 8$ are equal. The sum of the left-hand side is $\sum_{i=1}^{8} b_{i} c_{i}=[\phi]=1 / 2$. Similarly the right-hand sides give the sum $\phi-[\phi]=1-\frac{1}{2}$.

Let us now consider (3) with $\Phi$ given by (4)-(9). These elementary weights are all of degree 1 (in the sense used in [3]). Hence they are of the form

$$
\Phi=\left[\Phi^{\prime}\right]
$$

where $\Phi^{\prime}$ is an elementary weight of order $r-1$. Using a result in [3] we find

$$
\gamma=r \gamma^{\prime}
$$

Let us suppose further that $\Phi^{\prime}$ is of degree $s$ and

$$
\Phi^{\prime}=\left[\Phi_{1} \Phi_{2} \cdots \Phi_{s}\right]
$$

We now have

$$
\gamma^{\prime}=(r-1) \cdot \gamma_{1} \gamma_{2} \cdots \gamma_{s}
$$

Now we define an elementary weight $\Phi^{\prime \prime}$ with order $r$ and degree $s+1$ given by

$$
\Phi^{\prime \prime}=\left[\phi \Phi_{1} \Phi_{2} \cdots \Phi_{s}\right]
$$

so that

$$
\gamma^{\prime \prime}=r \gamma_{1} \gamma_{2} \cdots \gamma_{s}=\frac{r}{r-1} \gamma^{\prime}
$$

Suppose, finally that

$$
\Phi^{\prime}=\sum_{i=1}^{8} b_{i} \chi_{i}
$$

where $\chi_{1}, \chi_{2}, \cdots, \chi_{8}$ are functions of $a_{i j}, c_{i}(i, j=1,2, \cdots, 8)$ but not of $b_{1}, b_{2}, \cdots, b_{8}$.

By the definitions of $\Phi, \Phi^{\prime \prime}$ we have

$$
\begin{aligned}
\Phi & =\sum_{i, j=1}^{8} b_{i} a_{i j} \chi_{j}, \\
\Phi^{\prime \prime} & =\sum_{i=1}^{8} b_{i} c_{i} \chi_{i} .
\end{aligned}
$$

We now prove that $\Phi=1 / \gamma$ assuming the corresponding results for $\Phi^{\prime \prime}$ and $\Phi^{\prime}$. We have 


$$
\begin{aligned}
\Phi & =\sum_{i, j=1}^{8} b_{i} a_{i j} \chi_{i}=\sum_{j=1}^{8} b_{j}\left(1-c_{j}\right) \chi_{j}=\Phi^{\prime}-\Phi^{\prime \prime} \\
& =\frac{1}{\gamma^{\prime}}-\frac{1}{\gamma^{\prime \prime}}=1 /\left(r \gamma^{\prime}\right)=1 / \gamma .
\end{aligned}
$$

This result will now be applied to the elementary weights (4)-(9). For the case

we have

$$
\Phi=\left[{ }_{2}[\phi] \phi\right]_{2}
$$

$$
\boldsymbol{\Phi}^{\prime}=[[\phi] \phi], \quad \Phi^{\prime \prime}=\left[[\phi] \phi^{2}\right]
$$

which equal $1 / \gamma^{\prime}, 1 / \gamma^{\prime \prime}$ respectively as Huta's solution demands.

The elementary weights (5)-(9) are each of order 6 so if one of these is $\Phi$, then $\Phi^{\prime}$ is of order 5 and so equals $1 / \gamma^{\prime}$. Table 2 shows the form of $\Phi^{\prime \prime}$ in these cases.

TABLE 2

\begin{tabular}{cc}
\hline$\Phi$ & $\Phi^{\prime \prime}$ \\
\hline$\left[{ }_{2}[\phi] \phi^{2}\right]_{2}$ & {$\left[[\phi] \phi^{3}\right]$} \\
{$\left[\left[_{2}[\phi]^{2}\right]_{2}\right.$} & {$\left[[\phi]^{2} \phi\right]$} \\
{$\left[{ }_{2}\left[\phi^{2}\right] \phi\right]_{2}$} & {$\left[\left[\phi^{2}\right] \phi^{2}\right]$} \\
{$\left[_{2}[2 \phi]_{2} \phi\right]_{2}$} & {$\left[\left[_{3} \phi\right]_{2} \phi^{2}\right]$} \\
{$\left[{ }_{3}[\phi] \phi\right]_{3}$} & {$[[[\phi] \phi] \phi]$} \\
\hline
\end{tabular}

Comparing this table with table 1 we see that $\Phi^{\prime \prime}=1 / \gamma^{\prime \prime}$ is demanded by Huta's solution in all cases except $\Phi^{\prime \prime}=\left[\left[{ }_{2} \phi\right]_{2} \phi^{2}\right]$.

In this remaining case, $\Phi^{\prime \prime}$ is the partner of $\left[{ }_{2}[\phi] \phi^{2}\right]_{2}$ in Huta's formulation and the value of this we have just shown to be correct.

\section{References}

[1] Hut̆a, A., Une amélioration de la méthode de Runge-Kutta-Nyström pour la résolution numérique des équations différentielles du premier ordre, Acta Fac. Nat. Univ. Comenian. Math. 1 (1956), $201-224$.

[2] Huta, A., Contribution à la formule de sixième ordre dans la méthode de Runge-KuttaNyström, Acta Fac. Nat. Univ. Comenian. Math. 2 (1957), 21-24.

[3] Butcher, J. C., Coefficients for the study of Runge-Kutta integration processes, This Journal 3 (1963), 185-201.

University of Canterbury, Christchurch, New Zealand. 